\section{OP10 ASSESSING MODELS OF ACP IN PRIMARY CARE, THE META-LARC TRIAL: PART 4 CROSS US-CANADA ETHICS CHALLENGES AND EXPERIENCE}

${ }^{1} \mathrm{~A}$ Totten, ${ }^{1} \mathrm{~L}$ Michaels*, ${ }^{2} \mathrm{D}$ Caron, ${ }^{2} \mathrm{~S}$ Guay-Belanger, ${ }^{3} \mathrm{~K}$ Grant, ${ }^{3} \mathrm{M}$ Greiver. ${ }^{1}$ Oregon Health and Science Univ., Portland, USA; ${ }^{2}$ Universite Laval, Quebec, Canada; ${ }^{3}$ University of Toronto, Toronto, Canada

\subsection{6/spcare-2019-ACPICONGRESSABS.10}

Background We designed a collaborative, US-Canadian study to increase generalizability and explicitly consider potential for spread across different systems. Addressing ethical requirements for multiple Institutional Review Boards (IRBs) and Research Ethics Boards (REBs) presents challenges when conducting one trial in two countries.

Methods Our trial includes 42 primary care practices from practice-based research networks (PBRNs) in 5 US states and 2 Canadian provinces. A centralized, single IRB process was used in the US with Clinical and Translational Science Award authorization to the PI for the 5 IRBs to rely on one unassociated IRB. In Canada ethics oversight required different approaches in each province. Ontario used a partially centralized and an institutional REB while Quebec used a fully centralized REB. Similar study protocols were submitted to all IRBs/REBs. The joint coordinating center harmonized research workflows and procedures to respond to a variety of concerns and requests.

Results The number and nature of concerns requiring clarifications and modifications varied across the IRBs and REBs. The IRBs/REBs considered the trial to present different levels of risk and viewed the training, implementation and evaluation of ACP differently. There were differences regarding informed consent, survey language and data sharing. While the process and approvals took longer than expected and posed issues for consistency, addressing them facilitated development of a robust intervention and protocol.

Conclusion Differences in research ethics perspectives and procedures could be significant barriers to US-Canada research. We demonstrated that variation could be addressed, knowledge-sharing strengthened the project protocol, and future collaborations are possible.

\section{OP11 ASSESSING MODELS OF ACP IN PRIMARY CARE, THE META-LARC TRIAL: PART 5: PLANNING AND SUPPORTING IMPLEMENTATION}

${ }^{1} \mathrm{~A}$ Totten, ${ }^{1} \mathrm{D}$ Dorr*, ${ }^{1} \mathrm{~L}$ Michaels, ${ }^{1} \mathrm{~S}$ Izumi, ${ }^{1} \mathrm{~N}$ Shankle, ${ }^{2} \mathrm{~S}$ Strauss. ${ }^{1}$ Oregon Health and Science Univ., Portland, USA; ${ }^{2}$ University of Toronto, Toronto, Canada

\subsection{6/spcare-2019-ACPICONGRESSABS.11}

Background Implementation of ACP is challenging, requiring a multi-pronged approach in primary care. We sought to provide a toolkit that would facilitate practices' adoption of the Serious Illness Care Program, as a means of improving the quality of care and engaging patients in serious illness care discussions and planning.

Methods The joint coordinating center established a working committee to compile implementation resources. We used an iterative approach to identify key issues, materials, and discussion points necessary to engage practices in ACP implementation. We involved stakeholder groups representing patients, clinicians, practice facilitators, researchers, and informaticians. The group identified, adapted, and reached consensus on materials and approaches to facilitating ACP in primary care practices.

Results We identified potential implementation barriers, including knowledge, attitudes, workflow, health information technology constraints, and sensitivity of the topic to engaging practices in ACP. We gathered materials to address these barriers including checklists, adaptable templates for dissemination and documentation, and developed a guide to facilitate conversations with practices. The key topics included practice readiness, patient identification, use of prognostic algorithms, workflow enhancement, effective documentation, and sustainability. We are using the TiDier checklist to monitor implementation fidelity to the ACP models in the trial.

Conclusion We created a toolkit to support implementation of ACP in primary care practice that can be used by practice facilitators. It covers the major topics identified by stakeholders as essential for ACP implementation. We will evaluate and revise this, making an enhanced implementation guide available to the trial practices as well as to others.

\section{OP12 ASSESSING MODELS OF ACP IN PRIMARY CARE, THE META-LARC TRIAL: PART 6 DEVELOPING PATIENT- REPORTED MEASURES OF GOAL CONCORDANT CARE}

A Totten* ${ }^{*}$ D Dorr, L Michaels, S Izumi. Oregon Health and Science Univ., Portland, USA

\subsection{6/spcare-2019-ACPICONGRESSABS.12}

Background and aims Atient-reported goal concordance in serious illness care is an important primary outcome; however, no validated measure exists. As part of a trial of ACP, we sought to design and validate items for our trial that could form the basis for future measure development.

Methods To draft items we reviewed the literature for validated measures in goal attainment, shared decision-making, and concordance. We engaged stakeholders, including patients, providers, and researchers, to discuss what goal concordant care means, developed draft items, and reviewed these in two focus groups with patient and family advisors as well as with research teams from other funded advance care planning and palliative care projects.

Results Our draft instrument consists of 3 parts representing different approaches. The first part asks the patient whether their current care supports what is important to them and if any care received was unwanted. The second part is derived from the Life Preference Scale developed by Ariadne Labs. This asks patients to report on whether health care supports what they identify as their three most important goals. The third part includes process measures based on the content of serious illness conversations and asks patients if each step or activity happened.

Conclusion It is possible to develop a patient-reported measure of goal concordant care that has face validity based on measures of related or similar constructs and the input of a range of stakeholders. Validation and ongoing refinement of the measure will be conducted in parallel with the trial. 